\title{
Quantitative fragmented QRS has a good diagnostic value on myocardial fibrosis in hypertrophic obstructive cardiomyopathy based on clinical-pathological study
}

Xuanye Bi, Chengzhi Yang, Yunhu Song, Jiansong Yuan, Jingang Cui, Fenghuan Hu and Shubin Qiao* (D)

\begin{abstract}
Background: To investigate the relationship between fragmented QRS (fQRS) quantified by a new method and myocardial fibrosis (MF) and the diagnostic value of quantitative fQRS (Q-fQRS) to detect MF in hypertrophic obstructive cardiomyopathy (HOCM) patients based on histological validation.

Methods: We performed a retrospective study that included 69 patients with HOCM who underwent ventricular septal surgery. Nine individuals who died from accidents were studied as a control reference for the histological parameters. Septal myocardium samples were subjected to Masson's trichrome staining to quantify the collagen volume fraction (CVF). An fQRS pattern was defined as the presence of additional R waves or RSR', evidenced by notched R or S wave on electrocardiography (ECG). The Q-fQRS was quantified as the total amount of deflections in the QRS complex in all 12 routine ECG leads together. Cardiac magnetic resonance imaging was conducted, and late gadolinium enhancement (LGE) was measured at 2, 4, 6 and 8 standard deviations (SDs).

Results: Of the 69 patients, fQRS was documented in 38 (55.1\%) patients, the mean number of leads with fQRS was $3.7 \pm 1.6$, and the mean Q-fQRS was $17 \pm 7.2$. Compared with HOCM patients without fQRS, HOCM patients with fQRS had a higher CVF and more LGE at 6 SD $(P<0.001 ; P=0.040)$. Q-fQRS was correlated with CVF $(r=0.640, P<0.001)$, and Q-fQRS showed the best correlation with LGE measured at 8 SD $(r=0.379$, $P=0.002$ ). Multivariate regression analyses revealed that Q-fQRS was independently associated with the extent of CVF in HOCM patients after adjusting for age, sex, body surface area and the extent of LGE at 6 SD $(P<0.001)$. When the patients were divided into subgroups with normal CVF or high CVF according to the CVF in controls, Q-fQRS and LGE at 6SD showed similar diagnostic value in detecting patients with high CVF, with sensitivities of $66.7 \%$ vs $68.6 \%$, specificities of $76.7 \%$ vs $72.4 \%$, and accuracies of $71 \%$ vs $70.3 \%$.

Conclusions: HOCM patients with fQRS showed more extensive MF. Q-fQRS was an independent predictor for MF and had a good diagnostic value, with a sensitivity of $66.7 \%$ and specificity of $76.7 \%$, in identifying patients with higher fibrotic burden.
\end{abstract}

Keywords: Quantitative fragmented QRS, Myocadial fibrosis, Hypertrophic obstructive cardiomyopathy

\footnotetext{
* Correspondence: asbfw@sina.com

State Key Laboratory of Cardiovascular Disease, Fuwai Hospital, National Center for Cardiovascular Diseases, Chinese Academy of Medical Sciences and Peking Union Medical College, Beijing, China
}

(c) The Author(s). 2020 Open Access This article is licensed under a Creative Commons Attribution 4.0 International License, which permits use, sharing, adaptation, distribution and reproduction in any medium or format, as long as you give appropriate credit to the original author(s) and the source, provide a link to the Creative Commons licence, and indicate if changes were made. The images or other third party material in this article are included in the article's Creative Commons licence, unless indicated otherwise in a credit line to the material. If material is not included in the article's Creative Commons licence and your intended use is not permitted by statutory regulation or exceeds the permitted use, you will need to obtain permission directly from the copyright holder. To view a copy of this licence, visit http://creativecommons.org/licenses/by/4.0/ The Creative Commons Public Domain Dedication waiver (http://creativecommons.org/publicdomain/zero/1.0/) applies to the data made available in this article, unless otherwise stated in a credit line to the data. 


\section{Background}

Hypertrophic cardiomyopathy (HCM) is a common inherited cardiovascular disease that is characterized as myocardial fibrosis (MF) [1]. HCM patients with MF experience left ventricular (LV) adverse remodeling and have a poor prognosis, such as diastolic dysfunction, cardiovascular death, heart failure and fatal arrhythmia [2-6]. MF is a process of collagen deposition that is presently evaluated by endomyocardial biopsy, magnetic resonance imaging (MRI), or circulating biomarkers of collagen turnover. Each detection method has pros and cons.

Late gadolinium enhancement (LGE) is a standard approach for the assessment of MF because collagen deposition and excessive retention of gadolinium lead to enhancement on CMR images [7]. However, due to the limitations and significant costs of advanced techniques, CMR may not always be available. Fragmented QRS (fQRS) is becoming a novel electrocardiogram (ECG) finding and has potential use as a predictor of MF [8]. Qualitative analysis of fQRS has indicated that fQRS can detect myocardial fibrosis, as assessed by the extent of LGE during CMR imaging $[9,10]$. A recent study proposed a quantitative measurement of fQRS (Q-fQRS) and showed that Q-fQRS might be an early predictor of arrhythmogenic cardiomyopathy [11]. However, the association between Q-fQRS and MF and the diagnostic value of QfQRS remain unknown. Hence, we aimed to investigate the relationship between Q-fQRS and MF based on histologic validation in HOCM patients. Moreover, we also compared the diagnostic value of Q-fQRS to assess the extent of MF between LGE images and Q-fQRS.

\section{Method}

\section{Study population}

Sixty-nine consecutive HOCM patients with symptomatic left ventricular (LV) outflow tract obstruction who underwent surgical myectomy at Fuwai hospital,Beijing between 2015 and 2020 were retrospectively studied. All patients underwent a detailed cardiovascular evaluation, including medical history, clinical examination, 12-lead ECG, and CMR.

HCM was diagnosed by the criteria mentioned previously [12]...LV outflow obstruction was defined as an instantaneous peak doppler LV outflow tract gradient (LVOTG) $\geq 30 \mathrm{mmHg}$ at rest, or an exercised LVOTG $\geq 50 \mathrm{mmHg}$ [13]. Patients with obstructive coronary artery disease, history of alcohol septal ablation, history of surgery or trauma within the previous 6 months before the surgery, severe valvular disease,stages 3 to 5 chronic kidney disease (CKD),connective tissue disease and osteoarthropathy were excluded from the study.

Control myocardium from the LV septal wall was collected at autopsy of 9 individuals (6 males, 3 females, mean age $45.4 \pm 14.3$ years) who died from accidents, showing no signs of macroscopic or microscopic cardiac lesions.

\section{Electrocardiography}

A standard 12-lead ECG $(0.5-150 \mathrm{~Hz}, 25 \mathrm{~mm} / \mathrm{s}, 10 \mathrm{~mm} /$ $\mathrm{mV}$ ) was recorded in the supine position during quiet respiration before the surgeries. The fQRS was defined as previously described [14], as follows: in patients with QRS duration < 120 milliseconds, (1) an additional R wave ( $R$ prime), (2) notching in nadir of the $S$ wave, (3) notching of $\mathrm{R}$ wave, or (4) the presence of more than 1 $\mathrm{R}$ prime in 2 contiguous leads corresponding to the left ventricular (LV) segment; in patients with right or left bundle branch block (QRS duration $\geq 120$ milliseconds), (1) various RsR' pattern with $>2$ R', (2) $>2$ notches in the $\mathrm{R}$ wave, or (3) $>2$ notches in the downstroke or upstroke of the $\mathrm{S}$ wave, in 2 contiguous leads corresponding to the LV segment. The presence of fQRS in 2 contiguous anterior leads (V1-V5), lateral leads (I, aVL, and V6), or inferior leads (II, III, and aVF) was assigned to detect myocardial fibrosis in the anterior, lateral, or inferior segments, respectively. The Q-fQRS method quantifies the total amount of fragmentation in all 12 routine ECG leads together. The summation of absolute numbers of positive and negative deflection points in each first QRS complex of each ECG lead were labeled as a fQRS count value. The first deviation from the isoelectric line and the last transition from the last deflection to the iso-electric line were excluded [11].

\section{Echocardiography}

Transthoracic echocardiography was performed by an experienced cardio-sonographer using the Phillips iE33 Color Doppler Ultrasound System (Philips Healthcare, Andover, MA, USA). M-mode, twodimensional, and pulsed and continuous-wave Doppler studies were utilized in the standard evaluation according to the guidelines of the American Society of Echocardiography [15]. All patients underwent resting LVOT gradient measurements with continuous-wave Doppler echocardiography, while LVOT gradient after provocation was only determined in those with a resting LVOT gradient $<50 \mathrm{mmHg}$. The severity of mitral regurgitation was measured with flow Doppler imaging as a qualitative index (mild $=1$, moderate $=2$, moderate to severe $=3$, and severe $=4$ ) [16].

\section{Cardiac MRI}

CMR imaging was performed using a $1.5-\mathrm{T}$ speed clinical scanner (Siemens Medical Solutions, Erlangen, Germany) before ventricular septal myectomy. All images were acquired with an electrocardiographically gated breath-hold technique.

To evaluate functional parameters, electrocardiographic gating cine images were then acquired using a 
segmented, balanced, steady-state-free precession sequence. The images were acquired during multiple short breath holds (8-15 s). After scout images, cine imaging was performed in four chamber, three-chamber and two-chamber long- and short axis views with the following protocol: 6-mm-thick sections with a 2-mm gap between sections, repetition time, $2.7 \mathrm{~ms}$; eho time, $1.2 \mathrm{~ms}$; $70^{\circ}$ flip angle; temporal resolution, $40 \mathrm{~ms}$; field of view, $360 \times 315 \mathrm{~mm} 2$; matrix, $192 \times 162$ pixels; pixel size, $1.9 \times$ 1.3;slice thickness, $6 \mathrm{~mm}$.

All CMR images were analyzed using standard ventricular analysis software (Argus,VE36A; Siemens Medical Solutions). For all patients, wall thickness at the septal, posterior and LV end-diastolic dimensions were all determined in the short-axis view (at the midpapillary level). Epicardial and endocardial borders of the LV myocardium were manually traced during the whole cardiac phase on each cine short-axis image to obtain LV and RV enddiastolic and end-systolic volumes, ejection fractions, and myocardial mass. Myocardial mass was calculated by multiplying the volume of the myocardium calculated at end-diastole by the specific gravity of the myocardium $(1.05 \mathrm{~g} / \mathrm{ml})$. The end-diastolic volume index, end-systolic volume index, and mass index were indexed to body surface area. Late Gadolinium Enhanced (LGE) images were obtained $10-15 \mathrm{~min}$ after injection of $0.2 \mathrm{mmol} / \mathrm{Kg}$ gadolinium-DPTA.The extent of scarred myocardium was determined automatically by computer counting of all hyperenhanced pixels in the myocardium on each of the shortaxis images. Hyperenhanced pixels resembling LGE were defined as those with image intensities of 2SD, 4SD, $6 \mathrm{SD}$, and $8 \mathrm{SD}$ above the mean of image intensities in a remote myocardial region in the same image. A percentage of LGE was then generated by the ratio of total LGE mass to total LV mass.

\section{Cardiac surgery}

We applied extended septal myectomy evolving from the classic Morrow procedure. The hypertrophic ventricular septal leading to systolic anterior motion of the anterior mitral valve and LVOT obstruction was resected. The resection range in the long-axis direction started from approximately $4 \mathrm{~mm}$ below the aortic ring to the apex of the left ventricle beyond the bases of the papillary muscles. In the shortaxis direction, the myectomy started rightward to the nadir of the right aortic cusp and to the left and terminated near the mitral anterior commissure. Part of the LV anterior free wall detached to the ventricular septal causing LVOT narrowing may also need to be resected. Furthermore, the anomalous chordal attachments between the mitral valve leaflflets or papillary muscle and the ventricular septal were also excised. Additional surgery was performed based on expert consensus among the experienced cardiac surgeons. If intraoperative transoesophageal echocardiography detected a postoperative LVOT gradient $>30 \mathrm{mmHg}$ or more-than-moderate mitral valve regurgitation after weaning from cardiopulmonary bypass, reoperation was required.

\section{Histologic study}

The septal myocardium samples were immediately fixed in $10 \%$ buffered formalin and embedded in paraffin. The samples were sectioned and stained with Masson's trichrome staining for evaluating MF. Four images of every section were acquired with a projection microscope $(\times 200$;Fig. 3). Subsequent image analysis was

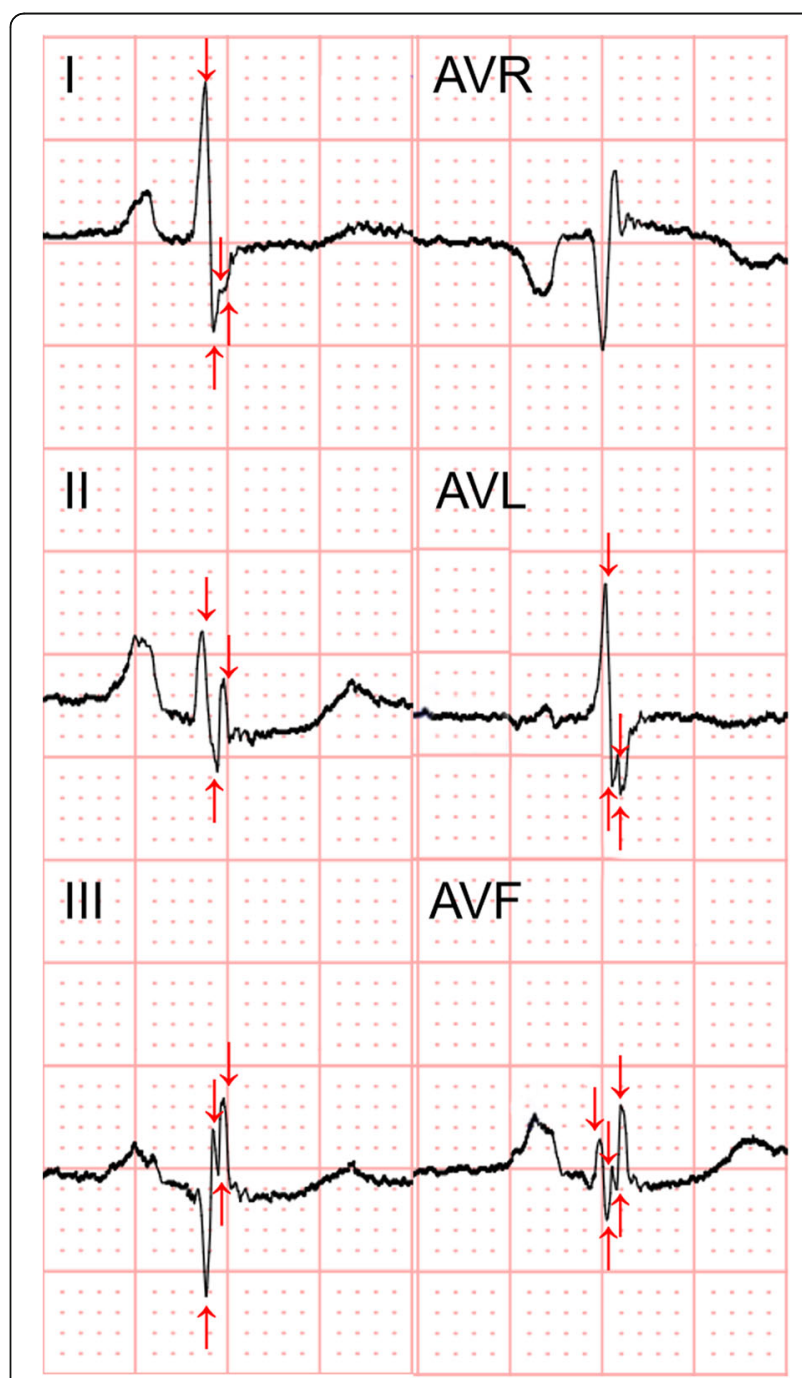

Fig. 1 Example of fagmented QRS. The different morphologies of fQRS are shown in this figure; notching of the R wave in leads II, III, and aVF, and notching in nadir of the $S$ wave in leads $\mathrm{I}$, aVL. The quantitative fQRS (QfQRS) counting is indicated by red arrows. Each arrow represents a positive or negative defflection counted as fractionated signal. Lead I, AVL, II, III, AVF have a Q-fQRS count being 4, 4,3, 4 and 5, respectively. The total Q-fQRS count in this figure add up to 20 
performed using Image-Pro Plus 6.0 image analysis software (Media Cybernetics Inc., Buckinghamshire, UK). To determine extent of myocardial fibrosis, which was expressed as collagen volume fraction (CVF,\%). CVF was calculated as the ratio of collagen-specific staining to the total area of the myocardium in each septal myocardium sample. The endocardium was excluded from analysis. This histological evaluation was performed by two well- trained cardio-pathologists without knowledge of which patient provided the tissue sections.

\section{Statistical analysis}

Continuous variables were expressed as a mean \pm standard deviation (SD) or median and interquartile range according to their distribution. Categorical variables were presented as percentage frequency. Differences between

Table 1 Baseline characteristics of 49 patients with HCM

\begin{tabular}{|c|c|c|c|c|}
\hline & $\begin{array}{l}\text { All patients } \\
(n=69)\end{array}$ & $\begin{array}{l}\text { Patients with HOCM } \\
\text { With fQRS } \\
(n=38)\end{array}$ & $\begin{array}{l}\text { Without fQRS } \\
(n=31)\end{array}$ & $\begin{array}{l}P \\
\text { value }\end{array}$ \\
\hline Age,years & $46.1 \pm 14.4$ & $43.7 \pm 15.6$ & $49 \pm 12.3$ & 0.129 \\
\hline Male, $\%$ & $43(62.3)$ & $22(57.9)$ & $21(67.7)$ & 0.401 \\
\hline Dyspnea,\% & $61(88.4)$ & $34(89.5)$ & $27(87.1)$ & 1 \\
\hline NYHA III/IV,\% & $22(31.9)$ & $13(34.2)$ & $9(29)$ & 0.646 \\
\hline History of syncope, $\%$ & $19(27.5)$ & $13(34.2)$ & $6(19.4)$ & 0.169 \\
\hline History of hypertension,\% & $15(21.7)$ & $6(15.8)$ & $9(29)$ & 0.185 \\
\hline History of diabetes mellitus, $\%$ & $6(8.7)$ & $4(10.5)$ & $2(6.5)$ & 0.867 \\
\hline Family history of HCM or SCD,\% & $5(7.2)$ & $2(5.3)$ & $3(9.7)$ & 0.813 \\
\hline NSVT,\% & $12(17.4)$ & $7(18.4)$ & $5(16.1)$ & 0.803 \\
\hline$A F, \%$ & $9(13)$ & $5(13.2)$ & $4(12.9)$ & 1 \\
\hline Bundle branch block,\% & $4(5.8)$ & $2(5.3)$ & $2(6.5)$ & 1 \\
\hline $\mathrm{ICD}, \%$ & $0(0)$ & $0(0)$ & $0(0)$ & 1 \\
\hline SAM, $\%$ & 68 (98.6) & $38(100)$ & $30(96.8)$ & 0.449 \\
\hline Mitral regurgitation & $2(1-2)$ & $2(1-3)$ & $2(1-2)$ & 0.373 \\
\hline Calcium antagonist, $\%$ & $25(36.2)$ & $11(28.9)$ & $14(45.2)$ & 0.163 \\
\hline Beta blocker,\% & $51(73.9)$ & $33(86.8)$ & $18(58.1)$ & 0.007 \\
\hline $\mathrm{ACEl} / \mathrm{ARB}, \%$ & $2(2.9)$ & $0(0)$ & $2(6.5)$ & 0.204 \\
\hline Diuretics, $\%$ & $24(34.8)$ & $11(28.9)$ & $13(41.9)$ & 0.26 \\
\hline \multicolumn{5}{|l|}{ CMR } \\
\hline Septal wall thickness,mm & $25(21-29)$ & $26(21.8-29)$ & $24(19-29)$ & 0.637 \\
\hline Left atrium diameter,mm & $41.8 \pm 7.9$ & $42 \pm 8.4$ & $41.5 \pm 7.3$ & 0.802 \\
\hline LV end-diastolic diameter,mm & $45.6 \pm 4.2$ & $46.1 \pm 4$ & $44.9 \pm 4.3$ & 0.236 \\
\hline LVMI,g/m² & $88.9(73.3-117.4)$ & $91.5(77.2-133.5)$ & $83(66.9-110)$ & 0.385 \\
\hline LVEF,\% & $64.8 \pm 9.6$ & $64.1 \pm 9.6$ & $65.8 \pm 9.8$ & 0.47 \\
\hline LVEDVI, $\mathrm{ml} / \mathrm{m}^{2}$ & $83.7 \pm 20.4$ & $87.7 \pm 23.2$ & $78.7 \pm 15.4$ & 0.069 \\
\hline LVESVI, $\mathrm{ml} / \mathrm{m}^{2}$ & $28.7(21.8-36.3)$ & $31.7(23.7-39.4)$ & $25.9(19.9-30.9)$ & 0.054 \\
\hline LGE at $2 S D, \%$ & $27.5(22.4-38.2)$ & $28(22.5-39.9)$ & $26.9(19-37.2)$ & 0.403 \\
\hline LGE at $4 \mathrm{SD}, \%$ & $12.8(6.9-19.1)$ & $14.4(9.5-21.9)$ & $11.9(5.8-16.7)$ & 0.067 \\
\hline LGE at $6 S D, \%$ & $6.2(1.9-8.7)$ & $6.7(3.9-10.8)$ & $4.2(1.6-7.5)$ & 0.04 \\
\hline LGE at $8 \mathrm{SD}, \%$ & $2.4(0.45-4.65)$ & $3.2(0.62-5.9)$ & $1.9(0.33-3.4)$ & 0.057 \\
\hline $\mathrm{BSA}, \mathrm{m}^{2}$ & $1.8(1.6-1.9)$ & $1.7(1.6-1.9)$ & $1.8(1.7-1.9)$ & 0.05 \\
\hline
\end{tabular}

Data are presented as the mean value SD ( $p$ values for 2-sided Student's t test) or median and interquartile ( $p$ values for Mann-Whitney test) or percentage of patients ( $\mathrm{p}$ values for chi-square test). Volumes are indexed to body surface area. $A C E I$ angiotensin-converting enzyme inhibitor, $A F$ atrial fibrillation, $A R B$ angiotensin receptor blocker, BSA body surface area, ICD implantable cardioverter-defibrillator, HCM hypertrophic cardiomyopathy, $L G E$ late gadolinium enhancement, LVEDVI Left ventricle end diastolic volume index, LVEF left ventricular ejection fraction, LVESVI Left ventricle end systolic volume index, LVMI left ventricle mass index, NYHA New York Heart Association, NSVT non-sustained ventricular tachycardia, SAM systolic anterior motion, SCD sudden cardiac death, SD standard deviation 
groups were compared with the use of the Student $t$ test (for parametric variables) and Mann-Whitney test (for nonparametric variables) for continuous variables. Categorical variables were compared with chi-square tests or Fisher's exact chi-square tests. The Spearman's correlation coefficient method was utilized to test for association between continuous variables. Patients were categorized in 2 subgroups according to values of CVF within (subgroup with normal CVF) and above (subgroup with high CVF) the upper limit of normality (established as mean $\pm 1.96 \mathrm{SD}$ obtained in control subjects and equal to 6.09\%). Receiver operating characteristic (ROC) analysis was plotted and the accuracy of the ROC curve was determined by measuring the area underneath it (AUC). Sensitivity, specifificity, positive predictive value (PPV), negative predictive value (NPV), and accuracy for the presence of fQRS were defined as previously reported [17]. Multivariate regression analysis was performed using stepwise of the following variables: age, sex, body surface area, Q-fQRS and LGE at 6SD to evaluate if these variables were independently associated with CVF, provided to have a $p<0.10$ in univariate analysis. This study assessed interobserver reproducibility for CVF by correlation and Bland-Altman analysis. All statistical analyses were conducted by using statistical software package SPSS Statistics V22.0. A value of $P<$ 0.05 was considered significant.

\section{Results}

Sixty-nine HOCM patients who underwent ventricular septal surgery were included, and LGE was assessed in 64 patients. Among the HOCM patients, the mean age was $46.1 \pm 14.4$ years; $43(62.3 \%)$ were male. fQRS was documented in 38 (55.1\%) patients. A representative ECG with fQRS is presented in Fig. 1. The mean number of leads with fQRS was $3.7 \pm 1.6$. fQRS was observed in the inferior $(32 / 38,84.2 \%)$, anterior $(10 / 38,26.3 \%)$, and lateral $(8 / 38,21.1 \%)$ leads.

The LV mass index, septal thickness, LV end-diastole volume index, LV ejection fraction were all comparable between HOCM patients with and without fQRS (all $P>$ 0.05). The extent of LGE at 6SD was higher in patients with fQRS than those without fQRS [6.7\%(3.9-10.8\%), vs $4.2 \%(1.6-7.5 \%), P=0.040]$. The baseline characteristics are listed in Table 1.

Representative images for MF were showed in Fig. 3. Whereas MF were slightly stained in controls (Fig. 3c), it exhibited different levels of expression in HOCM patients (Fig. 3d, e). Bland-Altman analysis showed that the mean difference was $0.1151 \%$ and the limit of agreement was 2.6361-2.8663\%(Fig. 2). Both pathologist showed good agreement with CVF values $(r=0.965, P<0.001)$. The mean CVF was higher in patients with HOCM than in controls $[6.52 \%$ (3.46-11.08\%) vs 3.49\% (2.90-4.53\%), $P=$ 0.015, Fig. 3a]. Patients with fQRS showed a higher CVF than patients without fQRS [9.92\% (5.89-13.41\%) vs 5.16\% (1.98-6.47\%), $p<0.001$; Fig. 3b].

In correlation analysis, CVF was correlated with LGE at 2, 4, 6 and 8 SDs $(\mathrm{r}=0.265, P=0.033 ; \mathrm{r}=0.485, P<$ $0.001 ; r=0.532, P<0.001 ; r=0.509, P<0.001$; Fig. $4 a)$. The number of leads with fQRS showed a positive correlation with CVF $(r=0.597 P<0.001$; Fig. $4 b)$. The number of leads with $\mathrm{fQRS}$ showed the best correlation with LGE measured at $8 \mathrm{SD}(r=0.306, P=0.0014)$. In addition, Q-fQRS was also correlated with CVF $(r=$ 0.640, $P<0.001$; Fig. $4 \mathrm{c}$ ), and Q-fQRS showed the best correlation with LGE measured at $8 \mathrm{SD}(r=0.379, P=$ 0.002). No further correlations were found between fQRS or Q-fQRS and LV structure parameters. Multivariate adjusted regression analyses revealed that Q-


Fig. 2 Bland-Altman analysis a and correlation $\mathbf{b}$ of CVF between 2 observers 
a

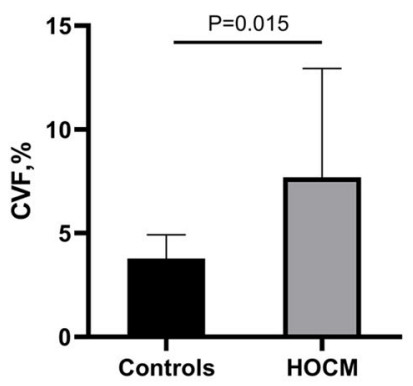

b



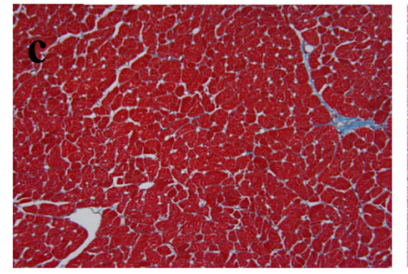
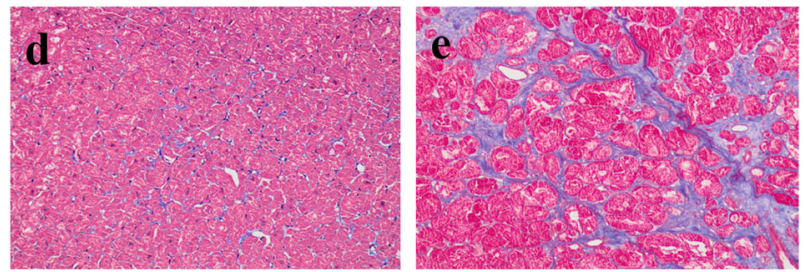

Fig. 3 Comparison of CVF in control subjects and HOCM patients and in fQRS(+) and fQRS(-) groups. HOCM patients had higher CVF value than controls $\mathbf{a}(P=0.015)$. CVF is higher in patients with fQRS than those without fQRS $\mathbf{b}(P<0.001)$. Myocardial fibrosis, stained in blue by Masson's trichrome staining $\mathbf{c}$, $\mathbf{d}$ and $\mathbf{e}$. The left panel correspond to mycardial fibrosis in controls $\mathbf{c}$, the middle and right panel correspond to mild and severe myocardial fibrosis in HOCM patients $\mathbf{d}$ and e.Magnification $\times 200$

fQRS was independently associated with the extent of CVF in HOCM patients after adjusting for age, sex, BSA, and the extent of LGE at 6 SD. See Table 2.

Taking into account the criteria mentioned previously, 30 patients exhibited normal CVF values [3.46\% (1.91$5.22 \%)$ ], and 39 patients exhibited high CVF values [subgroup with high CVF $10.48 \%$ (7.16-13.33\%)]. In the ROC analysis for the identification of patients with high CVF, the AUC, sensitivity and specificity of Q-fQRS, the number of leads showing fQRS and LGE at 6SD were
0.732 vs 0.715 vs $0.74,66.7 \%$ vs $59 \%$ vs 68.6 , and $76.7 \%$ vs $80 \%$ vs $72.4 \%$, respectively. See Table 3, Fig. 5 .

\section{Discussion}

In this study,we demonstrated the following points: 1 . HOCM patients with $\mathrm{fQRS}$ exhibited more extensive MF than those with normal QRS wave morphology; 2. QfQRS showed good correlation with the fibrotic state of the myocardium and was an independent predictor for MF in HOCM patients; and 3. compared with LGE at
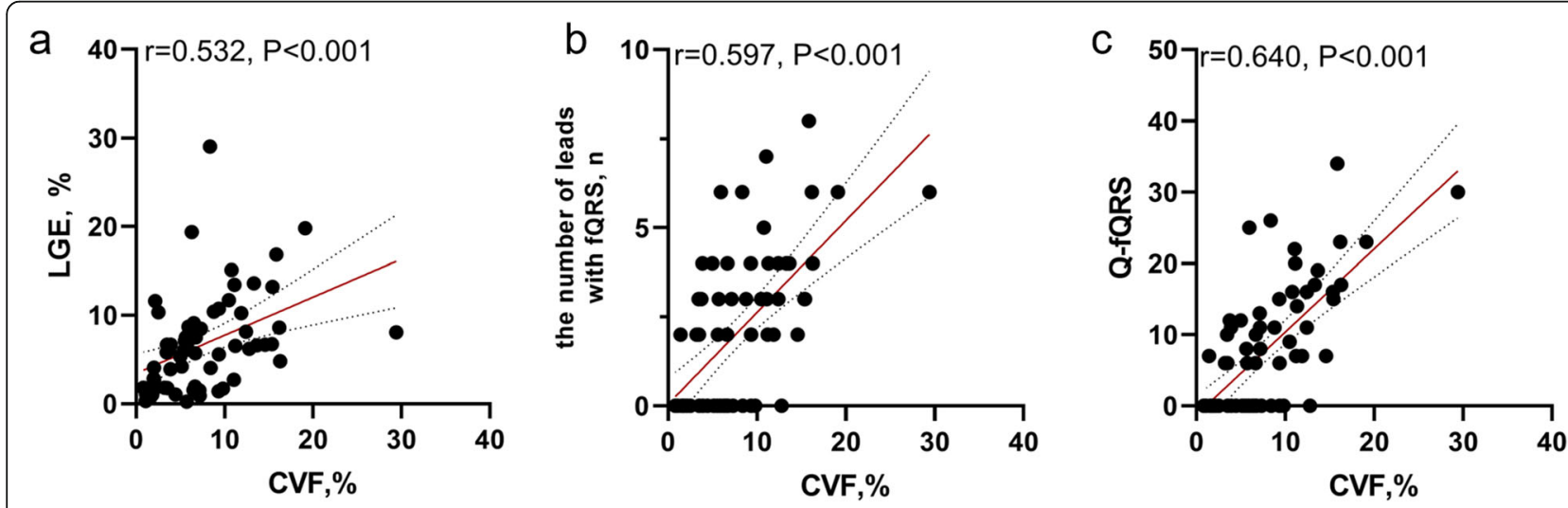

Fig. 4 Spearman's correlation analysis of CVF and clinic parameters. Significance was noted between CVF and LGE at 6SD a $(P<0.001)$, the number of lead with fQRS $\mathbf{b}(P<0.001)$ and $\mathrm{Q}-\mathrm{fQRS} \mathbf{c}(P<0.001)$ 
Table 2 Predictors of CVF in the HOCM Patients (Unadjusted and Multivariate Regression Analysis)

\begin{tabular}{llllll}
\hline Variable & Univariate & & & Multivariate & \\
\cline { 2 - 3 } & $\beta \pm S E$ & $P$ value & & $\beta \pm S E$ & $P$ value \\
\hline Age & $-0.043 \pm 0.044$ & 0.340 & & $-0.002 \pm 0.036$ & 0.953 \\
Sex & $-1.470 \pm 1.299$ & 0.262 & & $-1.246 \pm 1.060$ & 0.244 \\
BSA & $-6.015 \pm 3.341$ & 0.076 & & \\
Q-fQRS & $0.412 \pm 0.052$ & $<0.001$ & $0.408 \pm 0.067$ & $<0.001$ \\
LGE at 6 SD & $0.415 \pm 0.113$ & 0.001 & & \\
\hline
\end{tabular}

$B S A$ body surface area, CVF collagen volume fraction, $L G E$ late gadolinium, $Q$ $f Q R S$ quantitative fragmented $Q R S, S D$ standard deviation;

6SD, Q-fQRS showed a similar sensitivity and specificity in identifying patients with severe MF and could be used as an effective and cost-efficient predictor to evaluate fibrotic burden.

MF is one of the main pathological characteristics in HCM patients [17]. MF is associated with diastolic dysfunction, sudden cardiac death and heart failure $[4,18]$. Regarding the complex nature of the mechanisms of collagen deposition in HCM, including gene mutation, hemodynamic changes and microvascular rarefaction, the modality of MF varies and develops during the course of disease $[19,20]$. Unlike myocardial scars due to acute or chronic myocardial ischemia, MF can either be diffuse or present as multiple patches in HCM [21]. Previous studies showed that MF was mainly comprised of intravascular fibrosis and perivascular fibrosis in HOCM patients [22] and could be induced by old age, volume overload and pressure overload [23]. Although LGE was considered a standard assessment of MF, LGE was less reproducible in HCM than in other conditions. Because of extensive diffuse fibrosis, the areas of LGE were relatively discrete in diffusely abnormal myocardium. Thus, it was difficult to manually delineate the myocardium with a normal signal to set the normal threshold in HCM [24]. Despite good correlations between the extent of LGE and histological fibrosis, there is more to consider, and LGE cannot be simply equated with MF.

Twelve-lead ECG is a simple and cost-efficient tool. Patients with HCM frequently exhibit a wide spectrum of ECG abnormalities, especially in HCM patients with
LV outflow track obstruction [25]. fQRS was reported as a novel ECG marker that could be present in many cardiac diseases. The presence of fQRS was correlated with MF and could predict ventricular arrhythmia and poor prognosis $[8,26-28]$. A possible explanation for the formation of fQRS is that the increased presence of fibrotic tissue slows activation and results in inhomogeneous depolarization of the ventricles, which probably represents fragmentation in the QRS complex on surface 12lead ECG. Previous studies have found that fQRS was present in approximately $60 \%$ of HCM patients and was correlated with the extent of LGE, which is consistent with our studies [8, 26-28]. More importantly, we demonstrated the correlation between fQRS and the pathological fibrotic state in HOCM patients, supplementing our knowledge about fQRS.

However, fQRS is not limited to patients with cardiac diseases. fQRS is also present in almost $19.7 \%$ of subjects without a known cardiac disease and was not associated with increased mortality in these subjects. Although fQRS morphologies could be early markers of subclinical cardiac disease [29], it cannot be ruled out that some fQRS morphologies are benign normal variants, whereas other morphologies represent myocardial scarring. There is a need for a more detailed fQRS classification. Roudijk et al. reported a new approach to quantitate $\mathrm{fQRS}$ in patients with arrhythmogenic cardiomyopathy (ACM). With this method, they showed that both definite ACM patients and pathogenic variant carriers without an ACM diagnosis had a higher $\mathrm{Q}-\mathrm{fQRS}$ than the controls, which might indicate that increased Q-fQRS is an early sign of disease penetrance [11]. In our study, Q-fQRS was correlated with the extent of histological MF in HOCM patients. This indicated that patients with a higher Q-fQRS were more likely to have a higher fibrotic burden. Our study also showed that the diagnostic value of Q-fQRS was good with a sensitivity of $66.7 \%$ and specificity of $76.7 \%$ in detecting patients with severe MF. Compared with the qualitative definition of fQRS, QfQRS exhibited a higher sensitivity and accuracy, indicating that $\mathrm{Q}-\mathrm{fQRS}$ is an effective and cost-efficient tool to screen for a fibrotic state in HOCM patients.

One limitation of this study was that this was a retrospective study, however, we prospectively enrolled HOCM patients from 2015, and all patients were

Table 3 Predictive ability of each examination to discriminate HOCM patients with high CVF from those with normal CVF

\begin{tabular}{|c|c|c|c|c|c|c|c|c|}
\hline & AUC & $95 \% \mathrm{Cl}$ & Cutoff value & Sensitivity, $\%$ & Specificity, $\%$ & PPV, $\%$ & $\mathrm{NPV}, \%$ & Accuracy, $\%$ \\
\hline Q-fQRS & 0.747 & $0.611-0.838$ & 7 & 66.7 & 76.7 & 78.8 & 63.9 & 71 \\
\hline The numer of leads with fQRS & 0.729 & $0.594-0.825$ & 3 & 59 & 80 & 79.3 & 60 & 68.1 \\
\hline LGE at 6SD & 0.741 & $0.617-0.843$ & 6.22 & 68.6 & 72.4 & 75 & 65.6 & 70.3 \\
\hline
\end{tabular}

AUC area under curve, $C l$ confidence interval, CVF collagen volume fraction, NPV negative predictive value, $L G E$ late gadolinium, $P P V$ positive predictive value, $Q$ fQRS quantitative fragmented $Q R S, S D$ standard deviation; 


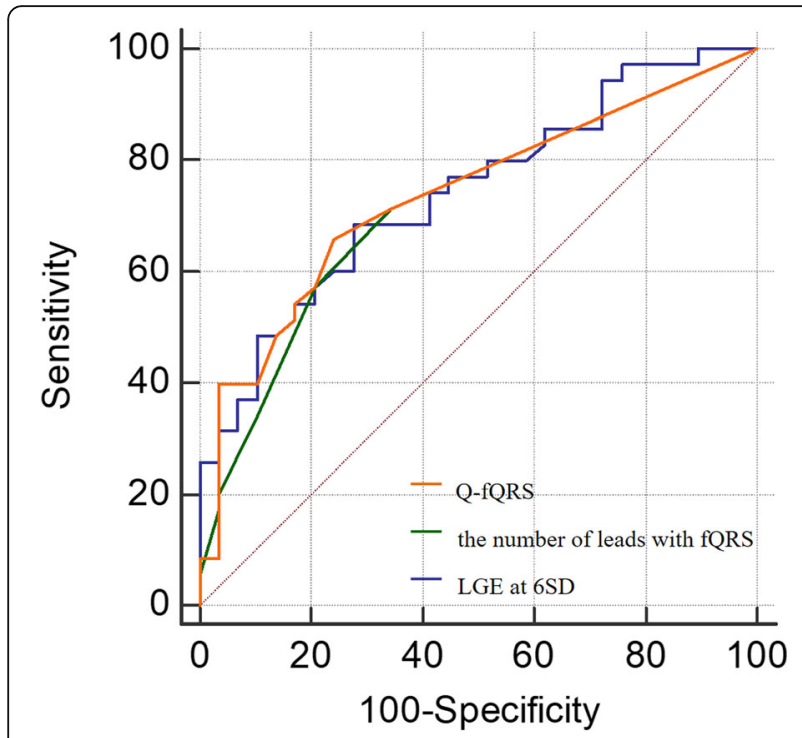

Fig. 5 Receiver operating analysis showing the diagnostic value for LGE at 6SD, the number of lead with fQRS and Q-fQRS to identify HOCM patients with high CVF

diagnosed and managed by protocol. Second,because the controls were coming from autopsies of 9 individuals who died from accidents, we had no access to their medical history. Third, sample size was small and restricted to HOCM, which needed to be validated in a larger HCM patient cohort. Fourth, our study limited to single samples of the septum obtained at surgical myectomy, so sampling in different regions might be essential to detect distribution of MF in HOCM patients. Fifth, the lack of long-term longitudinal follow up data prevented us from analyzing the prognostic effect in HOCM patients with high CVF and the influence of collagen accumulation during the development of HOCM.

\section{Conclusions}

In conclusion, HOCM patients with the presence of fQRS showed greater histological MF. Q-fQRS was an independent predictor to detect MF and had a good diagnostic value of $66.7 \%$ sensitivity and $76.7 \%$ specificity to identify patients with higher fibrotic burden.

\section{Abbreviations}

CVF: Collagen volume fraction; ECG: Electrocardiography; fQRS: Fragmented QRS; HOCM: Hypertrophic obstructive cardiomyopathy; LGE: Late gadolinium enhancement; LV: Left ventricular; MF: Myocardial fibrosis; NPV: Negative predictive value; PPV: Positive predictive value; fQRS: Quantitative fragmented QRS; ROC: Receiver operating characteristic; SD: Standard deviation

\section{Acknowledgements}

The authors thank the study patients for participating and the study personnel for their invaluable contribution. The authors also thank All staffs at the department of nuclear magnetic resonance for performing and assisting with the cardiovascular MRI analysis.

\section{Authors' contributions}

$\mathrm{XYB}, \mathrm{SBQ}$ and $\mathrm{CZY}$ contributed to the design of the study. XYB, YHS, JSY and FHH contributed to the analysis, while all authors(XYB, YHS, JSY, FHH, SBS, CZY) contributed to the interpretation of data. JGC contributed to the literarture review. XYB drafted the manuscript and SBQ and CZY contributed significantly to the preparation. All the authors critically revised the manuscript and gave final approval and agreed to be accountable for all aspects of the work, ensuring both its integrity and accuracy.

\section{Funding}

This study was supported by grants from the National Natural Science Foundation of China (nos. 81370327), which generously provided the necessary reagents but had no involvement in the design of the study or the collection, analysis and interpretation of the data or in writing of the manuscript.

\section{Availability of data and materials}

The datasets generated and analysed during the current study are not publicly available due to patient confidentiality but are available from the corresponding author on reasonable request.

\section{Ethics approval and consent to participate}

This study was carried out in accordance with the Declaration of Helsinki. Written informed consent was obtained from every patient. The study protocol was approved by the ethics committee of the Fuwai hospital (No. 2015-790).

\section{Consent for publication}

Not applicable.

\section{Competing interests}

The authors declare that they have no competing interests.

Received: 7 August 2019 Accepted: 16 June 2020

Published online: 18 June 2020

\section{References}

1. Varnava AM, Elliott PM, Sharma S, McKenna WJ, Davies MJ. Hypertrophic cardiomyopathy: the interrelation of disarray, fibrosis, and small vessel disease. Heart. 2000;84(5):476.

2. Basso C. Hypertrophic cardiomyopathy and sudden death in the young: pathologic evidence of myocardial ischemia. Hum Pathol. 2000;31(8):988-98.

3. Bruder O, Wagner A, Jensen CJ, Schneider S, Ong P, Kispert EM, Nassenstein K, Schlosser T, Sabin GV, Sechtem U, et al. Myocardial scar visualized by cardiovascular magnetic resonance imaging predicts major adverse events in patients with hypertrophic cardiomyopathy. J Am Coll Cardiol. 2010; 56(11):875-87.

4. Motoyasu M, Kurita T, Onishi K, Uemura S, Tanigawa T, Okinaka T, Takeda K, Nakano T, Ito M, Sakuma H. Correlation between late gadolinium enhancement and diastolic function in hypertrophic cardiomyopathy assessed by magnetic resonance imaging. Circ J. 2008;72(3):378-83.

5. O'Hanlon R, Grasso A, Roughton M, Moon JC, Clark S, Wage R, Webb J, Kulkarni M, Dawson D, Sulaibeekh L, et al. Prognostic significance of myocardial fibrosis in hypertrophic cardiomyopathy. J Am Coll Cardiol. 2010; 56(11):867-74

6. Shirani J, Pick R, Roberts WC, Maron BJ. Morphology and significance of the left ventricular collagen network in young patients with hypertrophic cardiomyopathy and sudden cardiac death. J Am Coll Cardiol. 2000;35(1): 36-44.

7. Karamitsos TD, Francis JM, Myerson S, Selvanayagam JB, Neubauer S. The role of cardiovascular magnetic resonance imaging in heart failure. J Am Coll Cardiol. 2009;54(15):1407-24.

8. Das MK, Suradi H, Maskoun W, Michael MA, Shen C, Peng J, Dandamudi G, Mahenthiran J. Fragmented wide QRS on a 12-lead ECG: a sign of myocardial scar and poor prognosis. Circ Arrhythm Electrophysiol. 2008; (4): 258-68.

9. Ratheendran AC, Subramanian M, Bhanu DK, Prabhu MA, Kannan R, Natarajan KU, Saritha Sekhar S, Thachathodiyil R, Harikrishnan MS, Pai PG. Fragmented QRS on electrocardiography as a predictor of myocardial scar in patients with hypertrophic cardiomyopathy. Acta Cardiol. 2019:1-5. 
10. Konno T, Hayashi K, Fujino N, Oka R, Nomura A, Nagata Y, Hodatsu A, Sakata K, Furusho H, Takamura M, et al. Electrocardiographic QRS fragmentation as a marker for myocardial fibrosis in hypertrophic cardiomyopathy. J Cardiovasc Electr. 2015:26(10):1081-7.

11. Roudijk RW, Bosman LP, van der Heijden JF, de Bakker JMT, Hauer RNW, van Tintelen JP, Asselbergs FW, Te Riele ASJM, Loh P. Quantitative approach to fragmented QRS in Arrhythmogenic cardiomyopathy: from disease towards asymptomatic carriers of pathogenic variants. J Clin Med. 2020;9(2):545.

12. Maron BJ, Maron MS. Hypertrophic cardiomyopathy. Lancet. 2013;381(9862): 242-55.

13. Gersh BJ, Maron BJ, Bonow RO, Dearani JA, Fifer MA, Link MS, Naidu SS, Nishimura RA, Ommen SR, Rakowski H, et al. 2011 ACCF/AHA guideline for the diagnosis and treatment of hypertrophic cardiomyopathy. J Am Coll Cardiol. 2011;58(25):e212-60.

14. Das MK, Maskoun W, Shen C, Michael MA, Suradi H, Desai M, Subbarao R, Bhakta D. Fragmented QRS on twelve-lead electrocardiogram predicts arrhythmic events in patients with ischemic and nonischemic cardiomyopathy. Heart Rhythm. 2010;7(1):74-80.

15. Naqueh SF, Bierig SM, Budoff MJ, Desai M, Dilsizian V, Eidem B, Goldstein SA, Hung J, Maron MS, Ommen SR, et al. American Society of Echocardiography clinical recommendations for multimodality cardiovascular imaging of patients with hypertrophic cardiomyopathy: endorsed by the American Society of Nuclear Cardiology, Society for Cardiovascular Magnetic Resonance, and society of cardiovascular computed tomography. J Am Soc Echocardiogr. 2011;24(5):473-98.

16. Jassal DS. Sustained improvement in left ventricular diastolic function after alcohol septal ablation for hypertrophic obstructive cardiomyopathy. Eur Heart J. 2006;27(15):1805-10

17. Grimes DA, Schulz KF. Uses and abuses of screening tests. LANCET. 2002; 359(9309):881-4

18. Ahn M, Kim J, Joung B, Lee M, Kim S. Prognostic implications of fragmented QRS and its relationship with delayed contrast-enhanced cardiovascular magnetic resonance imaging in patients with non-ischemic dilated cardiomyopathy. Int J Cardiol. 2013;167(4):1417-22.

19. Ho CY, López B, Coelho-Filho OR, Lakdawala NK, Cirino AL, Jarolim P, Kwong R, González A, Colan SD, Seidman JG, et al. Myocardial fibrosis as an early manifestation of hypertrophic cardiomyopathy. New Engl J Med. 2010; 363(6):552-63.

20. Mohammed SF, Hussain S, Mirzoyev SA, Edwards WD, Maleszewski JJ, Redfield MM. Coronary microvascular rarefaction and myocardial fibrosis in heart failure with preserved ejection fraction. Circulation. 2015;131(6):550-9.

21. Liu T, Song D, Dong J, Zhu P, Liu J, Liu W, Ma X, Zhao L, Ling S. Current understanding of the pathophysiology of myocardial fibrosis and its quantitative assessment in heart failure. Front Physiol. 2017;8.

22. Anderson $\mathrm{KR}$, Sutton MG, Lie JT. Histopathological types of cardiac fibrosis in myocardial disease. J Pathol. 1979;128(2):79-85.

23. Kong $P$, Christia $P$, Frangogiannis NG. The pathogenesis of cardiac fibrosis. Cell Mol Life Sci. 2014;71(4):549-74.

24. Flett AS, Hasleton J, Cook C, Hausenloy D, Quarta G, Ariti C, Muthurangu V, Moon JC. Evaluation of techniques for the quantification of myocardial scar of differing etiology using cardiac magnetic resonance. JACC Cardiovasc Imaging. 2011:4(2):150-6.

25. Savage DD, Seides SF, Clark CE, Henry WL, Maron BJ, Robinson FC, Epstein SE. Electrocardiographic findings in patients with obstructive and nonobstructive hypertrophic cardiomyopathy. Circulation. 1978;58(3 Pt 1): 402-8.

26. Das MK, Zipes DP. Fragmented QRS: a predictor of mortality and sudden cardiac death. Heart Rhythm. 2009;6(3):S8-S14.

27. Jain $\mathrm{R}$, Singh $\mathrm{R}$, Yamini $\mathrm{S}$, Das MK. Fragmented ECG as a risk marker in cardiovascular diseases. Curr Cardiol Rev. 2014;10(3):277-86

28. Ogura S, Nakamura K, Morita H, Toh N, Nakagawa K, Yoshida M, Watanabe A, Nishii N, Miyoshi T, Ito H. New appearance of fragmented QRS as a predictor of ventricular arrhythmic events in patients with hypertrophic cardiomyopathy. Circ J. 2020;84(3):487-94.

29. Terho HK, Tikkanen JT, Junttila JM, Anttonen O, Kenttä TV, Aro AL, Kerola T, Rissanen HA, Reunanen A, Huikuri HV. Prevalence and prognostic significance of fragmented QRS complex in middle-aged subjects with and without clinical or electrocardiographic evidence of cardiac disease. Am J Cardiol. 2014;114(1):141-7.

\section{Publisher's Note}

Springer Nature remains neutral with regard to jurisdictional claims in published maps and institutional affiliations.

\section{Ready to submit your research? Choose BMC and benefit from:}

- fast, convenient online submission

- thorough peer review by experienced researchers in your field

- rapid publication on acceptance

- support for research data, including large and complex data types

- gold Open Access which fosters wider collaboration and increased citations

- maximum visibility for your research: over $100 \mathrm{M}$ website views per year

At BMC, research is always in progress.

Learn more biomedcentral.com/submissions 